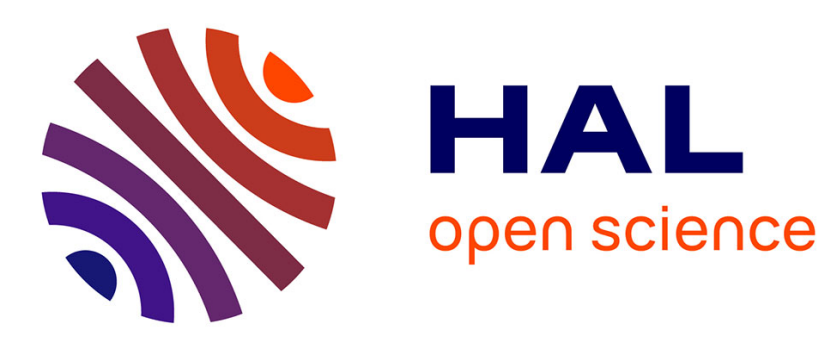

\title{
MEASUREMENT OF THERMODYNAMIC EQUILIBRIA BY CHROMATOGRAPHY
}

Ioannis Ignatiadis, Marie France Gonnord, Claire Vidal-Madjar

\section{To cite this version:}

Ioannis Ignatiadis, Marie France Gonnord, Claire Vidal-Madjar. MEASUREMENT OF THERMODYNAMIC EQUILIBRIA BY CHROMATOGRAPHY. Chromatographia, 1987, 23 (3), pp.215-219. 10.1007/BF02311484 . hal-00737634

\section{HAL Id: hal-00737634 \\ https://hal.science/hal-00737634}

Submitted on 2 Oct 2012

HAL is a multi-disciplinary open access archive for the deposit and dissemination of scientific research documents, whether they are published or not. The documents may come from teaching and research institutions in France or abroad, or from public or private research centers.
L'archive ouverte pluridisciplinaire HAL, est destinée au dépôt et à la diffusion de documents scientifiques de niveau recherche, publiés ou non, émanant des établissements d'enseignement et de recherche français ou étrangers, des laboratoires publics ou privés. 


\author{
I. Ignatiadis ${ }^{1}$ * / M. F. Gonnord 1 / C. Vidal-Madjar ${ }^{2}$ \\ Laboratoire de Chimie Analytique Physique, Ecole Polytechnique, UA 432 CNRS, Route de Saclay, 91128 Palaiseau Cedex, \\ France
}

\section{Key Words}

Gas chromatography

Liquid chromatography

Thermodynamic equilibria

Solution and adsorption thermodynamics

Gas adsorption isotherms

\section{Summary}

After a brief recall of the chromatographic principles, the different applications of gas chromatographic measurements of thermodynamic equilibria were reviewed. Gas and liquid chromatographies are now well known and elegant methods for measuring the physicochemical properties and phase equilibrium thermodynamic constants. Although fundamentally a dynamical method and mostly known as a powerful separation technique, chromatography can be schematized by a succession of equilibria of a chemical species partitioning between a mobile phase and a fixed liquid or solid stationary phase. It can be operated in either infinite dilution or finite concentration conditions and permits to collect a large number of data for calculating molecular interactions for solutes which are either rare or available at the trace level.

Gas chromatography permits the measurement of gas adsorption isotherms, gas-liquid equilibria, molecular diffusion and interaction virials. The modelization of successive partition equilibria occuring in the chromatographic column leads to rather simple expressions of differential enthalpy, entropy, free energy of adsorption or solution, variation of heat capacity, complexation constant, second virial coefficients, gas-solid and gas. liquid isotherms and also binary or ternary equilibria. The possibilities of High Performance-Liquid Chromatography to investigate adsorption from solutions and chemical equilibria are also discussed.

1 Present address: Département de Chimie, Groupe Masse-Chimie Théorique, Ecole Polytechnique, Route de Saclay, $91128 \mathrm{~Pa}$ laiseau Cedex, France.

2 Present address: Laboratoire de Physicochimie des Biopolymères, UM 27 CNRS, 2, rue Henri Dunant, 94320 Thiais Cedex, France.

\section{Introduction}

Fundamental aspects of the chromatographic technique render it a fast, efficient and appropriate method for measuring thermodynamic equilibria.

The application of gas chromatography to the measurement of chromatographic data has first been proposed by Littlewood, Phillips and Prince thirty years ago [1].

Although all the measurements of adsorption and solution thermodynamics can be made by other techniques, the chromatographic technique offers certain brilliant advantages:

(a) Measurements at infinite dilution are of first importance in the theory of equilibria. They can be obtained from classical techniques from often difficult extrapolations. They can be directly obtained from the elution of a small amount of pulse sample into a chroma. tographic column.

(b) Correlatively, as it allows to operate on very small amounts of both solute and solvent or sorbent, chromatography is also an extremely sensitive technique particularly valuable for solutes which are either rare or only available at the trace level.

(c) As it is also a separation technique, chromatography is a rapid technique: it permits to operate directly on either impure solutes or mixtures without requiring long pumping like static methods.

(d) Finally, as a highly efficient separator, the chromatographic column can be used for the theoretical interpretation of the separation of similar molecules exhibiting a very small difference in their free energies of adsorption or solution.

On the other hand, the chromatographic phenomenon is quite complex. Many by-effects such as adsorption or slow kinetics can appear requiring the use of rather complicated corrective terms in the data handling and interpretation. This sometimes compromises the credibility of the technique even if the measurements are highly reproducible. Last, another limitation of chromatography is its impossibility to operate with polar or very volatile station $v$ phase.

The chromatographic process involves the distribution of a solute between two phases: a gaseous or liquid mobile phase and a liquid or solid stationary phase. It is common to distinguish between gas chromatography (where the 
mobile phase is a gas), and liquid chromatography (where the moving phase is a liquid or a mixture of liquids), although the fundamentals of chromatography are applicable to both forms of chromatography. The retention theory of chromatography is rather simple when studying the interaction between two species and neglecting other interactions. Therefore, chromatography is generally used to study binary systems. However, multicomponent systems involving for example a stationary phase interacting with two components can also be studied by chromatography.

Thermodynamic measurements obtained from chromatography can be carried out in two concentration ranges: theory of linear chromatography is applicable at infinite dilution (when dealing with gas-liquid equilibria) or at zero surface coverage (for gas-solid equilibria). Non-linear chromatography describes equilibria at finite concentrations.

At infinite dilution, solute interactions are neglected. The equilibria thermodynamic properties are obtained by measuring the retention time of a pulse injection while the kinetic and transport processes are deduced from the elution peak profile.

At finite concentrations, the chromatographic technique is appropriate for the determination of distribution isotherms. Different methods can be used: frontal analysis, elution analysis at different concentrations and the "stepand-pulse" technique which consists in the analysis of the retention of a pulse on a steady plateau of constant concentration.

The applications of gas chromatography in physicochemical studies have been reviewed by several authors [2, 3]. The physicochemical applications of High Performance Liquid Chromatography (HPLC) have been the object of an extensive review [4]. A general survey of the thermodynamics of HPLC has recently been published [5]. This work will be limited to the study of the infinite dilution chromatography since measurements at finite concentration permits the determination of equilibrium isotherm in both gas-liquid [6], gas-adsorption [7] and liquid-adsorption $[8,9]$ systems.

\section{Theoretical Recalls}

The general relationship between the experimental chromatographic data (expressed through the retention volume) and the equilibrium distribution coefficient describing the solute partition between the mobile phase and the stationary phase has been established by Riedo and Kovats [10]:

$$
v_{R_{i}}=v_{m}\left[\left(\frac{\partial n_{i}}{\partial x_{i}}\right)_{0}-x_{i}^{o}\left(\frac{\partial n_{t}}{\partial x_{i}}\right)_{0}\right]
$$

where

$n_{i}=$ number of moles of solute $i$,

$x_{i}=$ molar fraction of solute $i$ in the mobile phase,

$n_{\mathbf{t}}=$ total number of moles in the system,

$v_{m}=$ average molar volume of the mobile phase.

The subscript ${ }^{\circ}$ refers to the initial composition of the eluant.
In gas-liquid or liquid-liquid chromatography, eq. (1) reduces to:

$$
V_{R}=V_{M}+V_{S} v_{m}\left(1-x_{i}^{0}\right)\left(\frac{\partial C_{i}^{S}}{\partial x_{i}}\right)_{0}
$$

where

$V_{S}=$ volume of the stationary phase,

$V_{M}=$ volume of the mobile phase.

This equation directly relates the experimental retention volume to the derivative of the equilibrium isotherm $C_{i}^{S}=f\left(x_{i}\right)$, at molar fraction $x_{i}^{0}$, if $C_{i}^{S}$ is taken as the concentration of the solute in the stationary phase. $V_{R}$ is there the retention volume of a pulse injected on a plate of molar fraction $x_{i}^{0}$ and corresponds to the retention volume observed in the "step-and-pulse" method which was first developed by Valentin and Guiochon [6]. At infinite dilution, $x_{i}^{0}=0$, and eq. (2) is reduced to its clas. sical expression for partition chromatography.

$$
v_{R}=v_{M}+V_{S} v_{m}\left(\frac{\partial C_{i}^{S}}{\partial x_{i}}\right)_{0}
$$

with a solid stationary phase, eq. (2) becomes:

$$
v_{R}=v_{M}+S v_{m}\left(\frac{\partial \Gamma_{i}}{\partial x_{i}}\right)_{0}
$$

where

$$
\begin{aligned}
& \Gamma_{i}=n^{S}{ }_{i} S=\text { Gibbs adsorption } \\
& n_{i}=\text { number of adsorbed molecules, } \\
& S=\text { total surface area of the adsorbent. }
\end{aligned}
$$

\section{Solution Thermodynamics from Gas-Liquid Chromatography}

\section{Activity Coefficients at Infinite Dilution}

The net retention volume $V_{N}=V_{R}-V_{M}$ is experimentally obtained from the difference between $t_{R}$, the transit time in the column of the retained compound, and $t_{m}$ the transit time of an unretained component.

$$
V_{N}=\left(t_{R}-t_{m}\right) \cdot J_{3}^{2} \cdot F
$$

where

$\mathrm{F}=$ column outlet flow rate,

$\mathrm{J}_{3}^{2}=$ a correction factor accounting for the pressure gradient $\left(P_{i}-P_{0}\right)$ in the column.

$$
J_{n}^{m}=\frac{n}{m} \frac{\left(P_{i} / P_{0}\right)^{m}-1}{\left(P_{i} / P_{0}\right)^{n}-1}
$$

It may be shown that the retention volume $V_{R}$ is related to the partition coefficient at infinite dilution $K_{1}$ defined as:

$$
K_{1}=\lim _{n_{1}^{\prime} \rightarrow 0} \frac{n_{1}^{1} V_{g}}{n_{1}^{g} V_{1}}
$$


where

$n_{1}^{g}$ and $n_{1}^{\prime}=$ numbers of the moles of solutes in the gaseous and liquid phases, respectively,

$V_{g}$ and $V_{1}=$ volumes of the gaseous and liquid phases, respectively.

$K_{1}$ is related to the activity coefficient at infinite dilution $\gamma^{\infty}$ and zero pressure through the expressions [11]:

$$
\operatorname{Ln} K_{1}=\operatorname{Ln} K_{1}(0)+\frac{2 B_{12}-v_{1}^{\infty}}{R T} \cdot P
$$

and

$$
\operatorname{Ln} K_{1}(0)=\operatorname{Ln} \frac{n_{1}^{l} R T}{\gamma^{\infty} p_{1}^{0} v_{1}^{0}}-\frac{B_{11}-v_{1}^{0}}{R T} p_{1}^{0}
$$

where $P$ is the system pressure, $B_{11}$ and $B_{12}$ are the second virial coefficients characterizing the interaction between two solute molecules and between one solute molecule and a carrier gas molecule, $v_{1}^{0}$ is the solute molar volume, $v_{1}^{\infty}$ the partial volume of the solute at infinite dilution in the stationary phase, $p_{1}^{0}$ is the saturation vapor pressure of the solute, $R$ is the gas constant and $T$ is the absolute temperature.

Considering the gas phase non ideality and the pressure drop through the column, one can calculate the retention volume of a solute in an ideal carrier gas as:

$$
\operatorname{Ln} V_{N}=\operatorname{Ln} K_{1}(0) V_{1}+\frac{B_{12}-V_{1}^{\infty}}{R T} P_{0} J_{3}^{4}+\ldots
$$

Eq. $(10)$ is applicable when using Nitrogen, Argon, Hydrogen or Helium as carrier gas, as little deviation from ideality is observed. From a plot of $\operatorname{Ln} V_{N}$ versus $P_{0} J_{3}^{4}$, one can calculate $\operatorname{Ln} K_{1}(0) V_{1}$ and thus obtain the activity coefficient at infinite dilution. Agreement between data obtained from chromatographic measurements and static methods is generally good [2]. When optimizing the support selection together with the amount of stationary phase and accounting for the pressure corrections, the accuracy in measuring the activity coefficient at infinite dilution by gas chromatography is about $1-2 \%$ [12].

Attention should be drawn on the fact that adsorption on the support and on the liquid stationary phase interface may contribute to the overall retention volume. It has been shown that liquid-surface adsorption occurs when a nonpolar solute interacts with a polar stationary phase but is negligible when dealing with non polar phases. Therefore, measurements of activity coefficients by gas chromatography are only limited to apolar solutes and apolar non volatile solvents in the limited temperature range suitable for carrying the experiments.

The partial molar enthalpy of mixing of the solute in the stationary phase can be obtained from the variation of $\gamma^{\infty}$ with temperature at constant pressure:

$$
\Delta H^{m}=R\left(\frac{\partial \operatorname{Ln} \gamma^{\infty}}{\partial(1 / T)}\right)_{P}
$$

The precision on $\Delta \mathrm{H}^{\mathrm{m}}$ measurement is about $300 \mathrm{~J}$ mole ${ }^{-1}$ for a $0.3 \%$ standard deviation in $\gamma$ measurement. Therefore, it is usually considered that it is more convenient to meas- ure $\Delta H^{m}$ from calorimetric than from chromatographic experiments.

The retention indices system which is widely used in analytical gas-liquid chromatography measures the retention of any compound relative to that of $n$-alcanes. The large amount of data that can be collected by gas chromatography has been exploited by correlating these parameters with the molecular structure of molecules [13].

\section{Mixed Second Virial Coefficients}

The gas-liquid chromatographic method permits the determination of the mixed second virial coefficient by varying the total pressure (cf eq. (10)). $B_{12}$ can be directly obtained from the slope of the straight line of the plot of $\operatorname{Ln} V_{N}$ versus $P_{0} J_{3}^{4}$. Eq. (10) remains valid when the carrier gas is insoluble in the stationary phase and the gas phase is ideal. Values of $B_{12}$ have been determined for hydrocarbons and various carrier gas systems [2].

\section{Adsorption Thermodynamics from Gas-Solid Chromatography}

At zero surface coverage the retention volume is related to Henry's adsorption constant $K_{H}$ which is the slope of the isotherm $n_{a}=f(p)$ at the origin $\left(n_{a}\right.$ is the number of adsorbed molecules per unit surface area of the adsorbent and $p$ the solute partial pressure).

$$
\operatorname{Ln}\left(V_{N} / A\right)=\operatorname{Ln} K_{H} R T+2 B_{2 D} n_{a}+2 B_{12} P_{O} J_{3}^{4} / R T
$$

Eq. (12) takes into account the non ideality of the gas phase [13] and the lateral interactions of the solute molecules in the adsorbed state (middle term). $A$ is the total surface area of the adsorbent.

The last term can be neglected when using a quasi ideal carrier gas like Helium.

The differential isosteric enthalpy of adsorption is obtained from the variation of $\mathrm{K}_{\mathrm{H}}$ with temperature:

$$
\Delta H_{s t}=-R \frac{d \operatorname{Ln} K_{H}}{d(1 / T)}
$$

and practically from the slope of the plot of $\operatorname{Ln}\left(V_{N} / A\right)$ versus $\frac{1}{T}$. Gas adsorption chromatography has been used in many instances to measure the thermodynamic functions of adsorption on various adsorbents [7]. Graphitized carbon black is a priviledged adsorbent for hydrocarbons as symmetrical peaks are observed. By carefully controlling the experimental parameters, the precision on $\Delta H_{s t}$ measurement is $50 \mathrm{cal} / \mathrm{mole}$ and the variation of the heat capacity during adsorption can be measured [14] and satisfactorily compared with static measurements when extrapolation at zero surface coverage from isotherm determination is possible. 


\section{Solution Thermodynamics from Liquid Chromatography}

Deriving an equilibrium partition coefficient from liquid chromatographic experiments is possible if the partitioning of the solute molecules between the liquid and stationary phases is rapid enough so that the departure from equilibrium summed over the whole column is negligible.

A detailed review [5] has been published, describing the thermodynamics of retention in reversed phase chromatography, adsorption chromatography, ion exchange chromatography, exclusion chromatography.

Considering the liquid-liquid chromatography retention mechanism as a partitioning of solutes between two bulk liquid solvents, Locke [4] has established the thermodynamics of retention at infinite dilution, relating retention volumes to solute activity coefficients. The activity coefficient in one phase can be determined if the activity in the other phase is known.

Several theories explaining the retention mechanisms in liquid-solid chromatography, with mixed solvent mobile phases, have been proposed [15]. It is shown that the retention volume is related in a simple manner with the volume fraction composition of the mixed solvent. The theory of chromatography with mixed liquid solvents developed by Riedo and Kovats [10] was applied to the determination of adsorption at the liquid-solid interface [16] and the possibility of defining and measuring a gas hold-up volume discussed.

\section{Complexes Equilibria Studies}

Complexes studies by gas-liquid chromatography are generally based on the measurement of the interaction between a solute and an additive to the stationary phase or directly of the interaction between the solute and the stationary phase.

The basic equations of chromatography are:

$$
\begin{array}{ll}
V_{N}^{*}=K_{1}^{*} V_{1} & \text { (absence of complexes) } \\
V_{N}=K_{1} V_{1} & \text { (presence of complexes) }
\end{array}
$$

The constants of the complexing equilibria are related to a theoretical expression giving $K_{1}$ as a function of $K_{1}^{*}$. Silver and other metal ligand complexes, charge transfer complexes and hydrogen bonding systems have been studied by gas-liquid chromatography [2].

The same principles for measuring complex equilibria are still valid in liquid chromatography [5] and have been used to measure complex associations of metals with molecules of biological interest. Horvath et al. [17] have demonstrated that HPLC with non polar stationary phases is a precise method for the evaluation of the stability constant of complexes formed in solution only in the case where the rate of equilibrium in the mobile phase is rapid.

\section{Reaction Kinetics}

The gas chromatographic columns is an "on-column reactor" which combines the function of a reactor and a separator. The theories assume an ideal chromatographic reactor where the distribution isotherm is linear and involve solutions for the mass balance equations. Kinetic data may be derived from the peak area ratios observed in the reaction chromatogram [2,3] or from measurements of the peak spreading [18].

Other techniques have been introduced in gas chromatography to study reaction kinetics: the stopped flow technique [19] uses the ability to move molecules at will under precise control through the chromatographic bed. Reactants, individual products and even impurities can be separated by the reversed flow technique which consists in reversing the direction of flow of the carrier gas from time to time [20].

The liquid chromatographic column can also be used as a reactor for kinetic studies [21].

\section{Diffusion Measurements}

The diffusion coefficient $D_{m}$ is measured from the band broadening of the elution peak as a function of carrier gas velocity. It is based on the height equivalent to a theoretical plate concept, $\mathrm{H}$, which is related to $\sigma_{t}$ the standard deviation of the elution peak in time units, the retention time $t_{R}$ and the column length. For an open tube column, $H$ is a function of the average mobile phase velocity $\bar{u}$ :

$$
H=L\left(\frac{\sigma_{t}}{t_{R}}\right)^{2}=2 D_{m}+\frac{r_{t}^{2} \bar{u}}{24 D_{m}}
$$

where

$2 r_{t}=$ internal diameter of the column.

The method and its application to the measurement of diffusion coefficients of vapors in various carrier gases has been reviewed by Maynard and Grushka [22]. Experimentally, equipment similar to the chromatographic one is required, including injection and detection devices.

The same method has been applied in liquid chromatography $[23,24]$ to determine diffusion coefficients at infinite dilution, mutual diffusion coefficients in liquids and self diffusion coefficients with a suitable marker.

\section{Conclusion}

In this quick review of the possible applications of chro. matography in thermodynamical equilibria measurements, the basic equations are given which allow further developments and the access to specific thermodynamic constants of the equilibria of solution or adsorption at infinite dilution, complexation constants, liquid interfacial effects, diffusion coefficients.

The measurement of thermodynamic data in gas - and liquid - phases by chromatography can favorably com. plete with static experiments in the infinite dilution range as direct measurements are carried on and extrapolations 
are unnecessary. On the experimental side, chromatography is a fast and sensitive technique for thermodynamic equilibria studies. It has however to be stressed that handling chromatographic data can sometimes be tedious and necessitate to account for secondary effects which lead to the introduction of correction factors which compromises the performances of the method.

\section{References}

[1] A. B. Littlewood, C.S. G. Phillips, D. T. Price, J. Chem. Soc. 1480 (1955).

[2] J.R. Conder, C.L. Young, in "Physicochemical Measurement by Gas Chromatography", Wiley, New York, 1979.

[3] J. Laub, R.L. Pecsok, in "Physicochemical Applications of Gas Chromatography". Wiley, New York, 1978.

[4] D.C. Locke, in "Advances in Chromatography", Vol. 8, J. C. Giddings, R. A. Keller, eds., Dekker, New York, 1969; pp. 47-89.

D.C. Locke, in "Advances in Chromatography", Vol. 14, J.C. Giddings, E. Grushka, J. Cazes, P. R. Brown, eds., Dekker, New York, 1976; pp. 87-198.

[5] C. Horvath, W. R. Melander, in "Chromatography, fundamentals and applications of chromatographic and electrophoretic methods. Part A. Fundamentals and techniques", Vol. 22 A, E. Heftmann, ed., J. of Chromatography Library, Elsevier, Amsterdam, 1983; pp. A27-A135.

[6] P. Valentin, G. Guiochon, J. Chromatogr. Sci. 14, 132 (1976).
[7] A. V. Kiselev, Y.I. Yashin, in "La chromatographie GasSolide". Masson, Paris, 1969.

[8] N. A. Chuduk, Y. A. Eltekov, A. V. Kiselev, J. Colloid Interface Sci. 84, 149 (1981).

[9] J.Jacobson, J. Frenz, C. Horvath, J. Chromatogr. 316, 69 (1984).

[10] F. Riedo, E. sz. Kovats, J. Chromatogr. 239, 1 (1982).

[11] D. H. Desty, A. Goldup, G. R. Luckhurst, W. T. Swanton, in "Gas Chromatography". M. Van Swaay, ed., Butterworths, London, 1962; pp. 67-83.

[12] M.W.P. Harbison, R. J. Laub, D.E. Martire, J. H. Purnell, P. S. Williams, J. Phys. Chem. 83, 1262 (1979).

[13] D. C. Locke, J. Phys. Chem. 69, 3768 (1965).

[14] C. Vidal-Madjar, M.F. Gonnord, M. Goedert, G. Guiochon, J. Phys. Chem. 79, 732 (1975).

[15] M. McCann, H. Purnell, C.A. Wellington, Faraday Soc. Symp. 15, 83 (1980).

[16] N. Le Ha, J. Unguaral, E. sz. Kovats, Anal. Chem. 54, 2410 [1982\}.

[17] C. Horvath, W. Melander, A. Nahum, J. Chromatogr. 186 371 (1979).

[18] J. Villermaux, J. Chromatogr. Sci. 12, $822(1974)$.

[19] K. F. Scott, C. S. G. Phillips, J. Catal. 51, 131 (1978).

[20] N.A. Katsanos, G. Karaiskakis, in "Advances in Chromatography", Vol. 24, J.C. Giddings, E. Grushka, J. Cazes, P. Brown, eds., Dekker, New York, 1984; pp. 125-180.

[21] M. K. Bolme, S. H. Langer, J. Phys. Chern. 87, 3363 (1983).

[22] V. R. Maynard, E. Grushka, in "Advances in Chromatography", Vol. 12, J.C. Giddings, E. Grushka, R. A. Keller, 1. Cazes, eds., Dekker, New York, 1975; pp. 99-140.

[23] E. Grushka, E. J. Kikta, J. Phys. Chem. 78, 2297 (1974).

[24] J. G. Atwood, J. Goldstein, J. Phys. Chem. 88, 1875 (1984).

Received: Feb. 2, 1987 Accepted: Feb. 12, 1987 